\title{
Postural Stability of Commercial Truck Drivers: Impact of Extended Durations of Whole-Body Vibration
}

\author{
Shaman Ahuja ${ }^{1}$, Jerry Davis ${ }^{1}$ and Lloyd Wade ${ }^{2}$ \\ ${ }^{1}$ Department of Industrial and Systems Engineering, Auburn University, AL \\ ${ }^{2}$ Department of Health, Exercise Science, and Recreational Management, University of \\ Mississippi, MS
}

Falls from non-moving vehicles constitute a significant portion of fall related fatal occupational injuries, with a yearly average of 48.7 fatal falls, from 1992 to 2002 . Whole-body vibration (WBV) has been thought to be a contributing factor to loss of balance, more specifically, falls while descending from a vehicle. The current research employed a "real-time" actual trucking environment, rather than vibration platforms in laboratories, as is typically the case. Nine (9) full-time, long haul truck drivers' drove over 3000 miles in total, while participating in this study. The protocol required postural stability testing prior to, and immediately following, each of three driving sessions of 2.5 hours during a single shift. Results indicate significant changes in postural stability as a result of exposure to extended durations of WBV. The results also showed an increase of sway $(\mathrm{cm})$ at each of the post-test measures compared to the pre-test, and an overall increase over the 8.5 hour shift, suggesting a time dependent increase over the course of the driving shift.

\section{INTRODUCTION}

Annually, the transportation industry is a leader in work related injuries, both fatal and nonfatal. According to the Bureau of Labor Statistics, in 2003, the Truck Transportation Industry (NAICS 484) reported 6.8 non-fatal injuries per 100 fulltime workers with 4.5 of these recordable cases requiring days away from work, job transfer, or restriction (Bureau of Labor Statistics: U.S. Department of Labor, 2003c; Bureau of Labor Statistics: U.S. Department of Labor, 2003b). Additionally, the Bureau of Labor Statistics (BLS) in 2003, truck transportation industry reported 517 fatal injuries, resulting from a myriad of events. Of these 517 fatalities, 17 were reported to be a result of falls from non-moving vehicles (BLS: U.S Department of Labor (DOL), 2003a). The BLS has documented falls from a non-moving vehicle as a significant portion of fall related fatal occupational injuries, with a yearly average of 48.7 fatal falls, from 1992 to 2002 (BLS: U.S DOL, 2003b). Specifically, long haul freight (LHF) trucking (NAICS 48412) accounted for approximately $70 \%$ of the fatal fall related injuries (BLS: U.S DOL, 2003a; United States Census Bureau, 2004, Chau, et al. 2004).

Though injuries ranging from transportation accidents to assaults have been reported, fall related injuries have been documented to be a significant percentage of injury statistics (Cornelius, et al. 1994;
Couch and Fraser 1981). Previous research suggests that fall related injuries (LHF trucking) are approximately 8 times more frequent during descent (egress) than those associated with ascending into the truck's cab (Nicholson and David 1985). Though specific causes of fall related incidents during egress are unclear, research has suggested that loss of balance is a contributing factor in such cases (Holbein and Redfern 1997; Shumway-Cook and Horak 1986).

Whole-body vibration (WBV) has been thought to be a contributing factor to loss of balance, more specifically, falls while descending from a vehicle (Nicholson and David 1985; Couch and Fraser 1981). The overall effect of WBV on the postural control system is relatively unclear, however it has been reported that the somatosensory and visual systems are affected by vibration (Martin, et al. 1980; Peli and Garcia-Perez 2003; Roll, et al. 1980). With prolonged exposure to WBV, the sensory information received from the somatosensory, vestibular, and/or visual systems could lead to postural instability, contributing to an increased risk of fall during egress of the vehicle. While previous research has been instrumental in determining the casual relationship of $\mathrm{WBV}$ and postural stability in simulated laboratory environments (uni-axial vibration) (Futatsuka et al., 1998; Martin et al., 1980; Cohen, 1979; Cornelius, 1994; Couch, 1981; Peli et al., 2003; Roll et al., 
1980); the lack of available real-time vibration exposure (tri-axial) in LHF truck drivers, leaves a theoretical void in the literature.

WBV is generated from a number of sources intrinsic to LHF truck operations such as varying road surface characteristics, rotating (translating) engine and drive-train components, and environmental factors (Hostens and Ramon 2003). Various factors that influence the transmission of vibration to the driver include size, mass, operating environment of the vehicular system, frequency and direction of input motion, seat characteristics, and individual differences (Jarvholm, et al. 2004; Martin, et al. 1980; Roll, et al. 1980; Hostens and Ramon 2003). Cohen et al. (1977) found that at $5 \mathrm{~Hz}$ the human body acts as an amplifier. Such resonance occurs when an object naturally strengthens a vibration at one or more particular frequencies, depending on the size and construction of the object (Plog and Quinlan 2002). Vehicular research has found that vibration frequencies as small as $1-2 \mathrm{~Hz}$ in the medio-lateral, and anterior-posterior direction, and/or 4-8 Hz inferior-superior directions, produced significant effects on the human body (Wasserman, et al. 1997). Moreover, it has been suggested that WBV in the frequency range of $0.5 \mathrm{~Hz}$ to $80 \mathrm{~Hz}$ may create chronic stresses and possible permanent damage to affected body parts (Hedge 2002).

Though a significant number of falls are reported annually in the LHF truck sector, there is a lack of research investigating the impact of WBV on the postural stability of LHF truck drivers. The purpose of the current research is to investigate the effect of WBV on the postural stability of LHF drivers, following vibration exposure. It is hypothesized that exposure to WBV will have a detrimental affect on the sensory system integration primarily associated with maintaining postural stability, and, this degradation in postural stability will increase with the extended duration of a typical driving shift.

\section{METHOD}

Nine (8 male, 1 female) full-time LHF truck drivers, employed by Auburn University's National Center for Asphalt Technology (NCAT) test track, took part in the study. Subjects (aged 42 to 74) provided informed consent prior to participating in the study. Exclusion criteria included; (1) the lack of ability to walk unassisted with normal gait, (2) chronic or acute lower limb injuries within the last year, (3) lower limb pathology, (4) a history of vestibular disorders, (5) lower extremity surgery, (6) eye disorders or surgery, (7) voluntary subject withdrawal, and/or (8) drug use.

The NCAT test track is a 1.9 mile oval track, designed to conduct accelerated pavement performance testing (APT) focused on applying a designed lifetime of truck traffic (10 to 15 years) in 2 years. The track consists of sections representing pavement typically found on United States highways. NCAT owns and operates five (5) Freightliner tractor-trailers (2000 Model), operating 2 shifts per day (0430-1300 \& 1400-2230), five days per week, around the calendar. The drivers are provided with top quality air ride seats to reduce fatigue. The average speed of trucks at the NCAT test track is $45 \mathrm{MPH}$. The trucks are representative of those seen on US roadways.

Postural stability measures were acquired utilizing a NeuroCom ${ }^{\circledR}$ Balance Master System ${ }^{\text {TM }}$ using the Modified Clinical Test of Sensory Interaction on Balance (mCTSIB). The mCTSIB protocol is composed of two trials (eyes open, eyes closed), each 10-seconds in duration, and has been widely utilized in research involving static tests of postural stability (Martin, et al. 1980; Wade, et al. 2004). The stability measures collected were Center of Force in the $\mathrm{x}$ and $\mathrm{y}$ directions (COFx and COFy) and Center of Gravity (COGx and COGy). Postural sway $(\mathrm{cm})$ was calculated using these measures and compared between the eyes open and eyes closed conditions.

HAVPro vibration equipment was utilized to measure tri-axial vibration $(\mathrm{Hz})$ over the course of a driving shift. This equipment has a sensitivity of 100 $\mathrm{mV} / \mathrm{g}$, with a RMS range of $0.01 \mathrm{~m} / \mathrm{s}^{2}$ to $5,000 \mathrm{~m} / \mathrm{s}^{2}$. The protocol required postural stability testing prior to, and immediately following, each of three driving sessions. Each driving shift ( 8.5 hours) was divided into three, 2.5 hour sessions, with a half-hour break between each driving session.

\section{Pre-Test}

The operator was instructed to drive to the testing station $(50 \mathrm{~m})$, egress from the truck, and step directly onto the force plate without any intervening contact with other surfaces. After testing, the operator was instructed to ascend back into the truck, and commence their 2.5 hour driving session. 


\section{Post-Test}

Following the 2.5 hour driving session, the subject was instructed to drive off the track to the post-test station, stop and egress from the truck directly onto the force plate without any intervening contact with other surfaces. When finished, operators ascended into the truck cab, and drove a short distance $(50 \mathrm{~m})$ to the parking area. Operators were scheduled for a 30 minute rest-break in between driving sessions. Following the break, the operator was once again pre-tested prior to the next 2.5 hour driving session. Following the final post-test session of the shift, drivers were dismissed.

\section{RESULTS}

RMS sway (cm) was analyzed in a 3 (Driving Session: 1,2,3) x 2 (Time: Pre-Exposure, PostExposure) x 2 (Eyes: Open, Closed) mixed ANOVA with repeated measures on Eyes to determine whether sway with eyes open or closed differed across driving routines. Independent ANOVAs were conducted on Anterior-Posterior (A/P) and MedioLateral $(\mathrm{M} / \mathrm{L})$ sway measures. For all analyses, a significance level of $\mathrm{a}=.05$ was set.

Analysis revealed a significant within subject's main effect for RMS A/P sway between driving sessions across time conditions for Eyes Open $\left(\mathrm{F}_{5,40}=3.24 ; \mathrm{p}<.05\right)$, however, revealed no significant differences in sway for Eyes Closed $\left(\mathrm{F}_{5,40}\right.$ $=.61, \mathrm{p}>.05)$ (Figure 1). Similarly, analysis revealed a significant within subject's main effect for RMS M/L sway between driving routines across time conditions for Eyes Open $\left(\mathrm{F}_{5,40}=2.90 ; \mathrm{p}<.05\right)$, but no significant differences in Eyes Closed conditions $\left(\mathrm{F}_{5,40}=1.88 ; \mathrm{p}>.05\right)$ (Figure 2$)$.

The results suggest that sway $(\mathrm{cm})$ measures during the 8.5-hour driving shift were: (a) greater in eyes open versus eyes closed testing conditions, (b) greater following a standard 2.5 hour driving session, (c) lower at the pre-test measures immediately following each of the 2 standard 30 minute breaks, and (d) greater at each of the post-test measures, with results indicating a time dependent increase over each driving shift.

\section{DISCUSSION}

Martin, et al. (1980) suggested that WBV in ranges less than $1 \mathrm{~Hz}$ and above $15 \mathrm{~Hz}$ has a deleterious effect on postural stability. Cornelius et al. (1994) indicated no statistical significant differences in postural stability following exposure to multiple ranges of WBV. It appears that some limitations associated with much of the previous research lie in the use of laboratory settings, specifically through the utilization of uni-axial vibration platforms. The current research implemented "real-time" tri-axial vibration employed in an actual trucking environment. The results of the current research indicate significant changes in postural stability as a result of exposure to extended durations of WBV.

Although, Cornelius et al. (1994) reported anecdotal evidence suggesting multiple vehicle operators indicated feelings of instability following extended durations (40-minutes) of operations in heavy mining vehicles; no statistical significant differences in postural stability measures were reported. The researchers suggested that factors which may affect postural stability that were not included in the study were multiple directions of vibration accelerations, varying axis of vibration acceleration, length of exposure, and environmental stressors as experienced in a "Real World" setting. In the current study, the various pavement sections on the NCAT test track represent US National highways, exposing the drivers to similar patterns of tri-axial whole body vibration. The results indicating tri-axial vibrations at extended durations ( 2.5 hours $\mathrm{x}$ 3 routines) showed significant differences in postural stability in LHF truck operators. The current study supports Cornelius et al. (1994) and Kjellberg and Wikstrom (1985) assertions, that there may be a cumulative effect of WBV over the course of an entire (8.5 hour) work shift. Although the driving pattern (2.5 hours on, $30 \mathrm{~min}$ off) is not typical of real world truck drivers, the current driving routine was fixed by NCAT to obtain optimum pavement testing.

Of particular interest in the current study is the differences in the eyes open and eyes closed testing conditions. The findings suggest that testing under the eyes open conditions resulted in significantly greater postural instability compared to eyes closed testing conditions. This suggests that the sensory information provided by the visual system was impacted in such a way as to reduce the utilization of the visual information in maintaining postural stability. Although, research has shown that available visual input can have an effect on the somatosensory and vestibular systems, and thus on postural control (Paulus and Brandt 1993; Paulus, et al. 1984; Redfern, et al. 2001; Wade, et al. 2004), it 
is worth noting that removing visual information (eyes closed) improved the operators ability to maintain postural control. It can be theorized that the extended duration of WBV contributed to involuntary eye vibration, in turn providing the operator with a false perception of environmental motion. This perception of environmental motion could increase postural sway through providing unreliable visual information, similar to that reported by Peli and Garcia-Perez (2003).

It can be concluded that postural stability may be impacted by exposure to WBV, similar to that experienced by LHF truck operators. Further, it is suggested that the relationship between WBV and postural stability may be a contributing factor in fall related accidents during egress from a LHF truck cab. Although, current findings suggest a casual relationship of WBV and postural instability, this study does not make insignificant the importance of confounding factors such as foot placement, equipment egress design, physiological and environmental elements that play a role in a possible fall incident. Future studies should be expanded to assess the effects WBV has on the visual system which may further improve the understanding of the role of sensory systems in postural control. Further research should look at the recovery aspect of exposure to WBV.

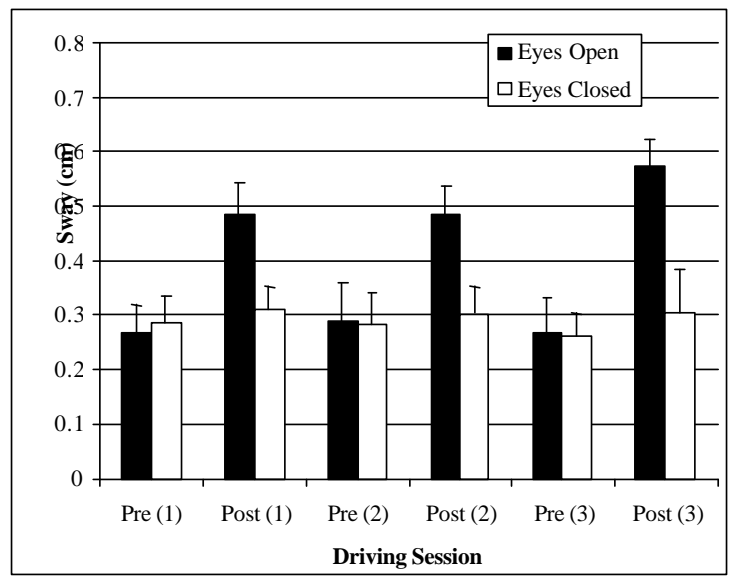

Figure 1. A/P sway $(\mathrm{cm})$ as a function of eye condition.

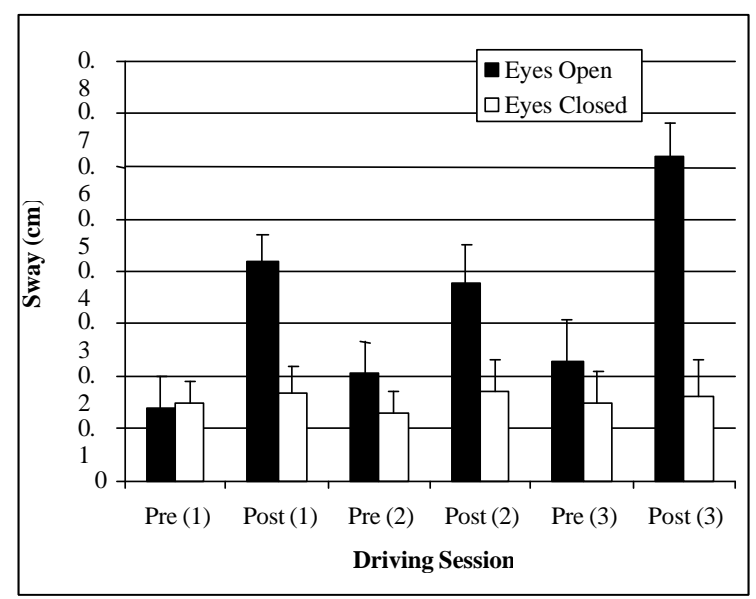

Figure2. M/L sway $(\mathrm{cm})$ as a function of eye condition.

\section{REFERENCES}

Bureau of Labor Statistics. "Fatal occupational injuries by industry and event or exposure." Census of Fatal Occupational Injuries (CFOI), Current and Revised Data, (2003a).

Bureau of Labor Statistics. "Fatal occupational injuries by selected characteristics: State of incident, employee status, sex, age, race, event or exposure, source, secondary source, nature, part of body, worker activity, location, occupation, and industry, 1992-2002" (revised final counts). Census of Fatal Occupational Injuries (CFOI), Current and Revised Data, (2003b).

Bureau of Labor Statistics. "Incidence rates of nonfatal occupational injuries and illnesses by industry and case types. OSHA recordable case rates, Current injury, illness, and fatality data, (2003c).

Chau, N., Gauchard, C.G., Siegfried, C., Benamghar, L., Dangelzer, J.L., Francais, M., Jacquin, R., Sourdot, A., Perrin, P.P., \& Mur, J.M. (2004). Relationships of job, age, and life conditions with the causes and severity of occupational injuries in construction workers. Int Arch Occup Environ Health, 77(1), 60-66.

Cohen, H.H., Wasserman, D.E., \& Hornung, R.W. (1977). Human performance and transmissibility under sinusoidal and mixed vertical vibration. Ergonomics, 20, 207-216.

Cornelius, K.M., Redfern, M.S., \& Steiner, L.J. (1994). Postural stability after whole body vibration exposure. International Journal of Industrial Ergonomics, 13(4), 343-351.

Couch, D.B., \& Fraser, T.M. (1981).Access systems of heavy construction vehicles: Parameters, problems and pointers. Applied Ergonomics, 12, 103-110. 
Futatsuka, M., Maeda, S., Inaoka, T., Nagano, M., Shono, M., \& Miyakita, T. (1998). Whole-body vibration and health effects in the agricultural machinery drivers. Industrial Health, 36(2), 12732.

Hedge, A. (2002). Whole body vibration. Cornell University.

http://ergo.human.cornell.edu/studentdownloads/ DEA350pdfs/Whole-body\%20Vibration.pdf.

Holbein, M.A., \& Redfern, M.S. (1997). Functional stability limits while holding loads in various positions. International Journal of Industrial Ergonomics, 19(5), 387-395.

Hostens, I., \& Ramon, H. (2003). Descriptive analysis of combine cabin vibrations and their effect on the human body. Journal of Sound and Vibration, 266(3), 453-464.

Jarvholm, B., Lundstrom, R., Malchau, H., Rehn, B., \& Vingard, E.(2004). Osteoarthritis in the hip and whole-body vibration in heavy vehicles. Int Arch Occup Environ Health, 77 (6), 424-426.

Kjellberg, A., \& Wikstrom, B.O. (1985). Whole-body vibration: Exposure time and acute effects-A review. Ergonomics, 28(3), 535-544.

Martin, B., Gauthier, G.M., Roll, J.P., Hugon, M., \& Harlay, F. (1980). Effects of whole-body vibrations on standing posture in man. Aviat Space Environ Med, 51(8), 778-787.

Nicholson, A.S., \& David, G.C. (1985). Slipping, tripping and falling accidents to delivery drivers. Ergonomics, 28(7), 977-991.

Paulus, W., \& Brandt, T. (1993). The role of visual motion in the stabilization of body posture. Rev Oculomot Res, 5, 405-417.

Paulus, W.M., Straube, A., \& Brandt, T. (1984). Visual stabilization of posture: Physiological stimulus characteristics and clinical aspects. Brain, 107, 1143-1163.

Peli, E., \& Garcia-Perez, M.A. (2003). Motion perception during involuntary eye vibration. Exp Brain Res, 149(4), 431-438.

Plog, B.A., \& Quinlan, P.J. (2002). Fundamentals of industrial hygiene. $\quad 5^{\text {th }}$ Ed. National Safety Council.

Redfern, M.S., Yardley, L., \& Bronstein, A.M. (2001). Visual influences on balance. Journal of Anxiety Disorders, 15, 81-94.

Roll, J.P., Martin, B., Gauthier, G.M., \& Maussa, I.F. (1980). Effects of whole-body vibration on spinal reflexes in man. Aviat Space Environ Med, 51(11), 1227-1233.

Shumway-Cook, A., \& Horak, F.B. (1986). Assessing the influence of sensory interaction of balance: Suggestion from the field. Physical Therapy, 66(10), 1548-50.

United States Census Bureau. (2004). 2002 NAICS Definitions. http://www.census.gov/epcd/naics02/naicod02.ht $\mathrm{m}$.

Wade, L.R., Weimar, W.H. \& Davis, J. (2004). Effect of personal protective eyewear on postural stability. Ergonomics, 47(15), 1614-1623.

Wasserman, D.E., Wilder, D. J., Pope, M.H., Magnusson, M., Aleksiev, A.R., \& Wasserman, J.F. (1997). Whole-body vibration exposure and occupational work-hardening. J Occup Environ Med, 39(5), 403-407. 